\title{
Reconciling new with old injury paradigms and the need to dig deeper - comment on Nigg et al.
}

\author{
Max R. Paquette ${ }^{1, *}$ \& Ross H. Miller ${ }^{2}$ \\ 1 School of Health Studies, University of Memphis, Memphis, TN, United States \\ 2 School of Public Health, Department of Kinesiology, University of Maryland, College Park, MD, United States \\ * Corresponding author: School of Health Studies, 308 Elma Roane Fieldhouse, University of Memphis, Memphis, Tennessee, \\ United States, 38152, Tel.: (1) 901-678-5025, Fax: (1) 901-678-3591 \\ Email: mrpqette@memphis.edu
}

\section{COMMENTARY}

\section{Article History:}

Submitted $16^{\text {th }}$ January 2018

Accepted $1^{\text {st }}$ March 2018

Published $18^{\text {th }}$ April 2018

Handling Editor:

Markus Tilp

Karl-Franzens-University Graz, Austria

Editor-in-Chief:

Martin Kopp

University of Innsbruck, Austria

\section{ABSTRACT}

In response to the target article by Nigg et al (2017) suggesting the need to shift towards new running injury paradigms, we comment on the need to continue investigating a variety of paradigms, new and old, and on poorly studied factors that necessitate the need to continue digging deeper in the pursuit of better prediction of injury development. Lastly, we argue that new and old paradigms can be reconciled under the more general paradigm that running injuries are most directly an issue of tissue adaptation.

Keywords:

Running - injury - movement - loading - adaptation

Citation:

Paquette, M. R. \& Miller, R. H. (2018): Reconciling new with old injury paradigms and the need to dig deeper - comment on Nigg et al. Current Issues in Sport Science, 3:105. doi: 10.15203/CISS_2018.105

This is a commentary on a CISS report article authored by Nigg, B. M., Mohr, M. \& Nigg, S. R. (2017). Muscle tuning and preferred movement path - a paradigm shift. Current Issues in Sport Science, 2:007. doi: 10.15203/CISS_2017.007

\section{Too early to abandon old paradigms}

\section{A. Conceptual framework}

An established injury paradigm should meet at least two sequential criteria: first, that the effect is real, and second, that the effect can be manipulated in a meaningful way. For example, to establish the impact forces paradigm, it should be shown that (i) impact forces are greater in injury-prone runners than in healthy runners, and (ii) reducing impact forces in injury-prone runners reduces their risk for injury.

\section{B. Usefulness of Incorrect Paradigms}

Under the criteria above, we agree that the evidence for the older running injury paradigms of impact forces and ankle pro- nation broadly explaining the mechanisms of running injury is unconvincing at this time. However, there seems to be little convincing evidence on any particular mechanism of injury in runners to date, so we suggest it is too early to discard any paradigms entirely. Much can be learned from pursuing paradigms that end up being wrong or only situationally correct. For example, the impact forces paradigm was supported in two recent prospective studies on recreational runners (Bredeweg, Kluitenberg, Bessem, \& Buist, 2013; Davis, Bowser, \& Mullineaux, 2016) but not in two others on competitive collegiate runners (Dudley, Pamukoff, Lynn, Kersey, \& Noffal, 2017; Kuhman, Paquette, Peel, \& Melcher, 2016). Rather than discarding this paradigm because all four studies did not support it, we stand to learn more by asking why the studies produced different levels of support: 
(i) Are there sources of false-positives in the former two studies?

(ii) Are there sources of false-negatives in the latter two studies?

(iii) Are there plausible mechanisms by which high loading rates would cause injury in recreational runners but not in competitive runners?

Answering these questions could lead to a revised impact forces paradigm, or conception of a new paradigm, even if the original paradigm is not strictly correct.

\section{Internal Loading and Tissue Adaptation}

Most running injury studies to date have used a "black box" framework where external loading is equated directly with injury risk (Figure 1a). Injuries in theory result more directly from internal loading above the thresholds of frequency and/or intensity for a tissue to experience positive remodeling (Hreljac, 2004). This paradigm is attractive conceptually because it provides a basis for explaining how more specific paradigms predict injuries mechanistically. To better understand the mechanisms of injury under any paradigm, the "black box" should be opened to more directly consider the influence of external loading on internal loading and resulting tissue injury (Figure 1b). The box could be opened conceptually by considering the mechanisms by which an external load may cause injury, by using animal/tissue-level models (e.g. (Loundagin, Schmidt, \& Edwards, 2017)), or by using computer models (e.g. (Wright, Neptune, van den Bogert, \& Nigg, 1998)). The proposed new paradigms would benefit from including explanations of how

A)

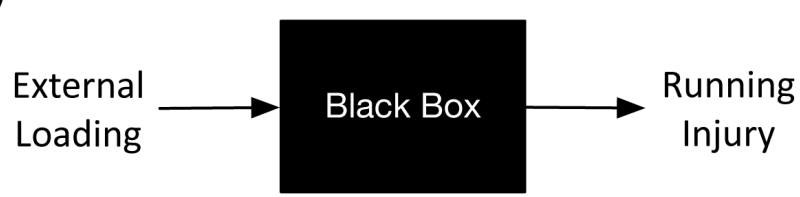

B)

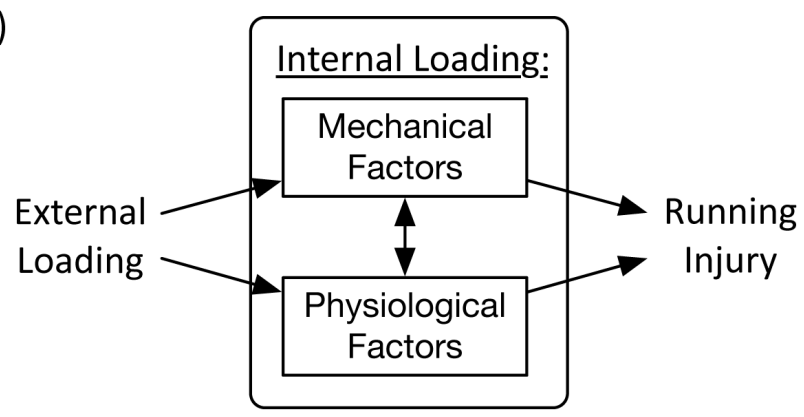

Figure 1: A) The "black box" framework between external loading and injury development, B) The necessary framework to understand the influence of external loading on internal loading, both mechanical and physiological, and resulting tissue injury. injuries occur mechanistically, how these explanations differ from the mechanisms suggested by older paradigms, and any testable predictions these explanations suggest.

\section{Why We Should Dig Deeper}

\section{A. Individual Training Backgrounds or "Functional Groups"}

Once we have a better understanding of internal loads associated with running, we can then consider poorly studied factors that can influence the relationship between external and internal loads which may explain curious phenomena in runners. For example, why can some people run with large external impact forces or loading rates without injury? Why can others accumulate massive internal loads without injury? Or why can some individuals run in any old shoe they want without injury? To date, we have limited answers to these questions as research often focuses on injured runners. However, we recommend that we shift our attention to those runners who can seemingly run as much as they want, sometimes in any shoes that they want, to better understand running injury paradigms.

One generally well accepted answer to the questions above is tissue adaptation as a result of gradual tissue loading and adequate recovery. The concept of safe and gradual increases in training workloads for injury reduction has received a considerable amount of attention in team sports (Gabbett, 2016; Hulin, Gabbett, Lawson, Caputi, \& Sampson, 2016). It is logical that safe and gradual workloads would be an effective strategy to reduce tissue loading and injury development in runners also. Such evidence in runners is still lacking or only anecdotal, but there is some evidence that runners of different training backgrounds and experience run with different movement patterns (Boyer, Freedman Silvernail, \& Hamill, 2014; Clermont, Osis, Phinyomark, \& Ferber, 2017; Maas, De Bie, Vanfleteren, Hoogkamer, \& Vanwanseele, 2017; Verheul, Clansey, \& Lake, 2017) that might subsequently alter internal loading of musculoskeletal tissue. Boyer et al (2014) observed differences in transverse pelvic, hip internal, hip and knee abduction and adduction, and frontal foot rotations between higher (>20 miles/week) and lower ( $<15$ miles/week) mileage runners. These transverse and frontal plane kinematic differences between training groups are suggested to potentially lower risks of knee injury development and may be the result of training-related neuromuscular adaptations as previously observed (Verheul, Clansey, \& Lake, 2017). Running ability/performance based on age-graded race times may also influence running kinematics. In general, greater magnitudes of three-dimensional pelvis, hip, knee and ankle angular positions during both swing and stances phases of recreational runners are observed compared to competitive runners (Clermont, Osis, Phinyomark, \& Ferber, 2017). Further, less and more experienced runners also appear to adjust their movement patterns differently in response to exhaustive running. Less experienced, or novice, runners run with larger kinematic adjustments in forward trunk lean and swing phase hip 
abduction compared to competitive runners during exhaustive running (Maas et al., 2017).

It therefore seems that the concepts of "functional groups", preferred movement paths and muscle tuning could be explained by the non-linear relationship between internal and external load, different physiological and biomechanical responses to chronic training workloads or exposures, and therefore, reconciling new and old paradigms.

\section{B. Factors That Need More Attention}

\section{Defining injuries}

Firstly, to have a fruitful conversation on the merits of different running injury paradigms, it is necessary to clearly define what we mean by "injury". A sensible definition for "overuse running injury" is:

A chronic imbalance in running-induced damage and recovery rates of the affected tissue, resulting in a deleterious change in tissue structure and/or function that limits training ability and/or performance.

This definition seems fairly uncontroversial and appears to have been used at least implicitly in most prior studies. However, large disparities exist in the literature on the criteria used to categorically define a runner as "injured", e.g. the duration of injury, the means of diagnosis, the severity of symptoms, etc. This lack of uniformity makes it difficult to compare results between studies. There is an evident need for a uniform definition of "injury" to ensure consistent diagnoses in research before we can discard injury paradigms or debate their merits.

\section{Baseline biomechanical and clinical screenings}

As recommended by Nigg et al (2017), more prospective studies are necessary to truly identify risk factors, biomechanical or otherwise, responsible for the development of running injuries. Current approaches for such prospective studies on running injuries consist of single-session baseline screenings including gait analyses, and clinical tests followed by survey periods in an attempt to identify predictive factors for the development of injuries. However, a critical flaw to this approach may be that these baseline screens are generally performed when runners are, acutely or chronically, non-fatigued. There is strong evidence for the fatigue- or exhaustion-related changes in running biomechanics especially in novice or recreational runners (Christina, White, \& Gilchrist, 2001; Maas et al., 2017; Mizrahi, Verbitsky, \& Isakov, 2000; Van Gheluwe \& Madsen, 1997). Different approaches for baseline screening procedures including biomechanical or clinical tests performed under acutely (i.e., after single bouts of exhaustive running) or chronically (i.e., after periods of heavy training) fatigued states could provide more sensitive baseline data to identify prospective injury development. Further, multiple testing sessions over a baseline period instead of a single testing session may be more sensitive for prediction of injury development. We acknowledge that such testing conditions or periods may not be practical for coaches and clinicians but may be necessary in the scientific pursuit of identifying risk factors for running-related injuries.

\section{Summary}

In summary, varying magnitudes of internal tissue loading and its resulting tissue adaptation, or lack thereof, to any given novel stimulus (e.g., footwear transitions, gait modifications, higher training intensities and/or volumes) is likely responsible for running injuries. We should consider poorly understood factors including baseline testing conditions in prospective injury studies before discarding old paradigms. Therefore, we must continue to explore both old (i.e., external loads, internal loads, tissue adaptation, non-linear relationship between external and internal loads) and new (i.e., preferred movement path, muscle tuning) running injury paradigms.

\section{Funding}

The authors have no funding or support to report.

\section{Competing Interests}

The authors have declared that no competing interests exist.

\section{Data Availability Statement}

All relevant data are within the paper.

\section{References}

Boyer, K. A., Freedman Silvernail, J., \& Hamill, J. (2014). The role of running mileage on coordination patterns in running. Journal of Applied Biomechanics, 30(5), 649-654. doi:10.1123/ jab.2013-0261

Bredeweg, S. W., Kluitenberg, B., Bessem, B., \& Buist, I. (2013). Differences in kinetic variables between injured and noninjured novice runners: A prospective cohort study. Journal of Science and Medicine in Sports, 16(3), 205-210. doi:10.1016/j. jsams.2012.08.002

Christina, K. A., White, S. C., \& Gilchrist, L. A. (2001). Effect of localized muscle fatigue on vertical ground reaction forces and ankle joint motion during running. Human Movement Science, 20(3), 257-276. doi:10.1177/0954411912447021

Clermont, C. A., Osis, S. T., Phinyomark, A., \& Ferber, R. (2017). Kinematic gait patterns in competitive and recreational runners. Journal of Applied Biomechanics, 33(4), 268-276. doi:10.1123/jab.2016-0218 
Davis, I. S., Bowser, B. J., \& Mullineaux, D. R. (2016). Greater vertical impact loading in female runners with medically diagnosed injuries: A prospective investigation. British Journal of Sports Medicine, 50(14), 887-892. doi:10.1136/bjsports-2015-094579

Dudley, R. I., Pamukoff, D. N., Lynn, S. K., Kersey, R. D., \& Noffal, G. J. (2017). A prospective comparison of lower extremity kinematics and kinetics between injured and non-injured collegiate cross country runners. Human Movement Science, 52, 197-202. doi:10.1016/j.humov.2017.02.007

Gabbett, T. J. (2016). The training-injury prevention paradox: Should athletes be training smarter and harder? British Journal of Sports Medicine, 50(5), 273-280. doi:10.1136/bjsports-2015-095788

Hreljac, A. (2004). Impact and overuse injuries in runners. Medicine \& Science in Sports \& Exercise, 36(5), 845-849.

Hulin, B. T., Gabbett, T. J., Lawson, D. W., Caputi, P., \& Sampson, J. A. (2016). The acute:Chronic workload ratio predicts injury: High chronic workload may decrease injury risk in elite rugby league players. British Journal of Sports Medicine, 50(4), 231-236. doi:10.1136/bjsports-2015-094817

Kuhman, D. J., Paquette, M. R., Peel, S. A., \& Melcher, D. A. (2016). Comparison of ankle kinematics and ground reaction forces between prospectively injured and uninjured collegiate cross country runners. Human Movement Science, 47, 9-15. doi:10.1016/j.humov.2016.01.013

Loundagin, L.L., Schmidt, T., \& Edwards, W.B. (2017). Is the mechanical fatigue of bone influenced more by the impact or active phase of running? Journal of Biomechanical Engineering, 140(3). doi:10.1115/1.4038288

Maas, E., De Bie, J., Vanfleteren, R., Hoogkamer, W., \& Vanwanseele, B. (2017). Novice runners show greater changes in kinematics with fatigue compared with competitive runners. Sports Biomechanics, 1-11. doi:10.1080/14763141.2017.134 7193

Mizrahi, J., Verbitsky, O., \& Isakov, E. (2000). Fatigue-related loading imbalance on the shank in running: A possible factor in stress fractures. Annals of Biomedical Engineering, 28(4), 463-469.

Nigg, B.M., Mohr, M., \& Nigg, S.R. (2017). Muscle tuning and preferred movement path - a paradigm shift. Current Issues in Sport Science, 2. doi:10.15203/CISS_2017.007

Van Gheluwe, B., \& Madsen, C. (1997). Frontal rearfoot kinematics in running prior to volitional exhaustion. Journal of Applied Biomechanics, 13, 66-75. doi:10.1123/jab.13.1.66

Verheul, J., Clansey, A. C., \& Lake, M. J. (2017). Adjustments with running speed reveal neuromuscular adaptations during landing associated with high mileage running training. Journal of Applied Physiology (1985), 122(3), 653-665. doi:10.1152/japplphysiol.00801.2016

Wright, I. C., Neptune, R. R., van Den Bogert, A. J., \& Nigg, B. M. (1998). Passive regulation of impact forces in heel-toe running. Clinical Biomechanics (Bristol, Avon), 13(7), 521-531. doi:10.1016/S0268-0033(98)00025-4 